\title{
Serum carotenoids as biomarkers of fruit and vegetable consumption in the New York Women's Health Study
}

\author{
Anne Linda van Kappel ${ }^{1, *}$, Jean-Paul Steghens ${ }^{2}$, Anne Zeleniuch-Jacquotte ${ }^{3,4}$, Véronique \\ Chajès $^{1}$, Paolo Toniolo ${ }^{3,4,5}$ and Elio Riboli ${ }^{1}$ \\ 'Unit of Nutrition and Cancer, International Agency for Research on Cancer, 150 cours Albert-Thomas, F-69372 \\ Lyon Cedex 08, France: ${ }^{2}$ Laboratoire de Biochimie C, Hôpital Edouard Herriot, 5 place d'Arsonval, \\ F-69437 Lyon Cedex 03, France: ${ }^{3}$ Nelson Institute of Environmental Medicine, New York University School of \\ Medicine, 650 First Avenue, 5th Floor, New York, NY 10016, USA: ${ }^{4}$ Kaplan Comprehensive Cancer Center, \\ New York University School of Medicine, 550 First Avenue, New York, NY 10016, USA: ${ }^{5}$ Division of Epidemiology, \\ Department of Obstetrics and Gynecology, New York University School of Medicine, 550 First Avenue, \\ Room NB 9E2, New York, NY 10016, USA
}

Submitted 27 July 2000: Accepted 27 November 2000

\begin{abstract}
Objective: To investigate the usefulness of serum carotenoids as biomarkers of fruit and vegetable consumption.

Design: Reproducibility study on three repeat measurements of serum carotenoids. Correlation analysis of carotenoids and dietary food intake, and regression analysis of potential predictive parameters for serum carotenoid levels.

Setting: New York, USA.

Subjects: Women participating in the New York Women's Health Study, a prospective study of sex hormones, diet and breast cancer. Forty-eight women with three repeat blood samples and 302 women having a blood sample and a dietary history questionnaire (including 47 subjects from the reproducibility study).

Results: Serum carotenoid concentrations were highly reproducible between oneand two-year repeat samples. Estimated fruit and vegetable consumption was positively correlated with serum carotenoid concentrations but correlation coefficients were low. Consumption of fruit was predictive for serum levels of betacarotene, alpha-carotene and beta-cryptoxanthin, while vegetable consumption was predictive for serum beta-carotene, lutein, zeaxanthin and lycopene. Serum concentrations of cholesterol and triglycerides were predictive for serum carotenoids but adjustment for their levels had little or no influence on the correlation between fruit and vegetable consumption and serum carotenoid concentrations.

Conclusions: One single serum measurement of alpha-carotene, beta-carotene and lutein can accurately rank subjects according to their usual serum level. Serum concentrations, however, correlate only moderately with estimated dietary intake of fruits or vegetables and should therefore be used with caution as biomarkers of fruit and vegetable intake.
\end{abstract}

Keywords

Carotenoids

Serum

Reproducibility

Consumption of fruit and vegetables
There is a strong and consistent pattern showing that diets high in vegetables and fruits decrease the risk of many cancers and other degenerative diseases such as cardiovascular diseases ${ }^{1}$. Carotenoids are natural pigments generally found in fruits and vegetables. More than 600 naturally occurring carotenoids are known today ${ }^{2,3}$, and analysis of serum and human breast milk has shown that about 20 carotenoids derived from fruits and vegetables may be absorbed and metabolised by humans ${ }^{4,5}$. Foods and supplements that contain carotenoids are the only source for humans, as carotenoids are synthesised only by plants.

It has been suggested that blood concentrations of carotenoids may be integrated biochemical markers of vegetable, fruit and supplement intake. Many epidemiological studies, however, have relied on single measurements of carotenoids in blood without clear evidence that a single serum or plasma measurement is adequate to characterise an individual long-term level, relative to other subjects in the population being studied. In fact, a 
measured concentration may have many exogenous and endogenous determinants, such as intake, absorption, distribution over body compartments or tissues, metabolism, excretion or the analytical method. High intraindividual, relative to inter-individual, variability may cause misclassification of a substantial number of individuals when a single measurement is used. Also, the association between a marker and the true dietary intake level or a disease parameter will be attenuated by these additional random variations. Several investigators $^{6,7}$ have suggested that multiple samples from each individual are required to identify the true serum concentration at an acceptable confidence level. The number of samples required can then be reduced by minimising inter-assay variability and identifying factors that influence circulating levels.

The purpose of this study was to investigate the quality and usefulness of serum carotenoids as a biochemical marker of fruit and vegetable consumption in a population of women from New York who were participating in a prospective study on hormones, diet and breast cancer. The reproducibility of serum concentrations and the influence of parameters on circulating blood levels and on intake-blood level relationships were investigated in the same population in order to evaluate carotenoids in blood as markers of nutritional status and as markers of dietary intake of fruits and vegetables.

\section{Subjects and methods}

\section{Subjects}

Study subjects were participants in a prospective cohort study on hormones, diet and cancer: the New York University Women's Health Study (NYUWHS). Between March 1985 and June 1991, 14,275 women aged 34-65 years who visited a large breast cancer screening centre in New York City (Guttman Breast Diagnostic Institute) agreed to donate $30 \mathrm{ml}$ of peripheral venous blood and to answer a lifestyle and medical history questionnaire, as well as a self-administered food-frequency questionnaire (FFQ). No recommendations were made about diet or use of supplements. Each time a cohort member returned to the clinic for subsequent annual mammographic screening, she was asked to donate an additional blood sample. Details concerning subject recruitment are published elsewhere $^{8,9}$.

For the reproducibility study, 48 cohort members (24 pre-menopausal, 24 post-menopausal), who had three blood samples taken at almost exactly one-year intervals, were selected. One measurement was missing for betacryptoxanthin and three for alpha-carotene. When the first blood sample was taken, the subjects were on average 52.6 years old (SD 7.5, range 38-67) and had an average body mass index (BMI) of $24.1 \mathrm{~kg} \mathrm{~m}^{-2}$ (SD 3.1 , range 19.5-34.6). Women taking vitamin supplements were not excluded, but at the time of the first blood sample only two women consumed a beta-carotene supplement daily.

For the validation study, results from first measurements in these 48 women were pooled with results from 270 control subjects from a nested case-control study on breast cancer ${ }^{10}$. Eleven subjects were excluded because of incomplete food intake questionnaires, four because of missing anthropometric measurements and, for one subject who was included in both the reproducibility and the breast cancer study, only results from the latter study were maintained. As a result, 302 subjects were included in the validation study, all having complete data for diet, anthropometry and laboratory measurements of serum carotenoids. Only six subjects indicated use of a beta-carotene supplement, five of them daily. At the time of blood collection, women were on average 51.7 years old (SD 8.3, range 35-65), had an average BMI of $25.2 \mathrm{~kg} \mathrm{~m}^{-2}$ (SD 4.9, range 16.9-55.1), 144 women said they were pre-menopausal and 158 post-menopausal. Cholesterol and triglyceride measurements were available for 228 subjects.

\section{Blood collection}

A 30-ml peripheral venous blood sample was drawn between 9 a.m. and 3 p.m. from non-fasting women and immediately centrifuged at $2000 \mathrm{~g}$ for $15 \mathrm{~min}$ at room temperature. The serum was partitioned in 1-ml aliquots in screw-cap tubes and immediately stored at $-80^{\circ} \mathrm{C}$ until biochemical analysis. The samples for the present study were shipped under dry ice to our laboratories in Lyon, France and stored at $-80^{\circ} \mathrm{C}$ upon arrival.

\section{Laboratory analyses}

On the day of analysis, samples were thawed at room temperature and protected from light during the entire experiment. Assays for levels of carotenoids, retinol and alpha-tocopherol were done with reversed-phase highperformance liquid chromatography (HPLC) ${ }^{11}$ on a Hewlett Packard 1100 system with ultraviolet + visible (UV + VIS) light sources and diode array detection (Hewlett Packard, les Ullis, France). For the reproducibility study, the three serum samples from each subject were analysed within the same batch. Control samples from a pool were included every day, completely blind for the laboratory technicians. Between-day coefficients of variation $(\mathrm{CV})$ varied from a maximum of $6.8 \%$ (betacarotene) to a minimum of $4.5 \%$ (beta-cryptoxanthin), except for zeaxanthin which had a CV of $11.4 \%$. Total cholesterol and triglycerides $(N=228)$ (expressed as mmol $1^{-1}$ ) were measured using an enzymatic method at $37^{\circ} \mathrm{C}$ with colorimetric detection on an automated system (Hitachi 747, Roche, France).

\section{Estimated food intake}

Dietary food intake was estimated using a self-administered, semi-quantitative diet questionnaire consisting of 
Table 1 Reproducibility of carotenoid concentrations in three serum samples taken with one-year intervals $(N=48)$

\begin{tabular}{|c|c|c|c|c|c|c|}
\hline & $\rho(1-2) \dagger$ & $\rho(2-3) \dagger$ & $\rho(1-3) \dagger$ & $\rho$ (Intraclass) $\ddagger$ & Ratio W/B§ & Number of measurements required \\
\hline Beta-carotene & 0.86 & 0.85 & 0.86 & 0.84 & 0.19 & 0.8 \\
\hline Alpha-carotene & $0.81^{\star}$ & $0.83^{\star}$ & $0.69^{\star}$ & $0.83^{\star}$ & 0.21 & 0.9 \\
\hline Lutein & 0.79 & 0.75 & 0.81 & 0.80 & 0.25 & 1.1 \\
\hline Zeaxanthin & 0.79 & 0.71 & 0.71 & 0.63 & 0.58 & 2.5 \\
\hline Beta-cryptoxanthin & $0.78^{*}$ & $0.75^{\star}$ & 0.75 & $0.58^{*}$ & 0.73 & 3.1 \\
\hline Lycopene & 0.61 & 0.62 & 0.55 & 0.63 & 0.59 & 2.5 \\
\hline
\end{tabular}

${ }^{*} N=47$.

† Spearman rank-order correlation between two measurements.

$\ddagger$ Intraclass correlation calculated as the ratio of the within-subject variance to the total variance.

$\S$ Ratio of within (W) to between (B) subject variability.

I Number of measurements needed to correctly rank at least $80 \%$ of individuals in the extreme thirds of the distribution.

70 items of typical American foods, adapted from a questionnaire developed and validated at the National Cancer Institute ${ }^{12}$.

\section{Statistics}

Statistical analysis was performed with SAS statistical software for PC release 8.0 (SAS Institute, Inc., Cary, NC). $t$-Tests were used to compare carotenoid means per visit, after $\log _{\mathrm{e}}$ transformation to reduce departures from the normal distribution. Statistical significance was estimated using 95\% confidence intervals and two-sided $P$-values. Spearman's rank correlation coefficients were calculated between repeat measurements. Adjustment for serum total cholesterol, serum triglycerides and body mass index (BMI, $\mathrm{kg} \mathrm{m}^{-2}$ ) did not improve rank-order correlations, so crude correlations are presented here. The intra-class correlation coefficient, which is the proportion of the total variance due to between-subject variance, was used to quantify the reproducibility of the serum carotenoid concentrations. Restricted maximum likelihood techniques in SAS PROC VARCOMP were used to estimate the intra-class correlation coefficients. The number of separate days $(D)$ necessary to rank individuals correctly according to their long-term analyte levels was computed using the formula proposed by Nelson et al. ${ }^{13}: D=$ $\left(r^{2} / 1-r^{2}\right)\left(S_{\mathrm{W}}^{2} / S_{\mathrm{B}}^{2}\right)$, where $r$ is the unobservable correlation between the measured and the true underlying values for an individual for a day, and $S_{\mathrm{W}}^{2}$ and $\mathrm{S}_{\mathrm{B}}^{2}$ are the observed within- and between-subject variances. As explained by Cooney et $a l^{6}{ }^{6}$, a chosen value of $r=0.9$ would mean that at least $80 \%$ of subjects in the extreme thirds of the distribution would be correctly classified, and $<1 \%$ would be grossly misclassified in the incorrect extreme thirds. The number of days (D) required increases with the precision desired on the measurement (r).

Spearman's rank-order correlation coefficients were calculated to investigate the associations between serum carotenoids and dietary intake of different food groups, BMI, serum cholesterol and triglycerides. Correlations with dietary intakes were adjusted for BMI. The influence of different dietary factors, serum cholesterol and triglycerides, age at blood collection, menstrual status, body mass index (BMI, $\mathrm{kg} \mathrm{m}^{-2}$ ), season and storage time were investigated using multiple regression analysis in GLM procedures with the serum carotenoid as the dependent variable. The backward elimination procedure was used to estimate sum of squares for each independent variable (denominated as Type III estimates in SAS). The ratio of each variable's sum of squares over the sum of squares of the model (partial $r^{2}$ ) indicates the proportion of the variation that is explained by the model that can be attributed to each independent variable. Date of blood collection was used as an approximate to storage time before laboratory analysis.

\section{Results}

\section{Reproducibility}

Average levels of carotenoids, retinol and alpha-tocopherol did not differ between first and second samples. However, third sample levels of beta-cryptoxanthin $(P=$ 0.02), lutein $(P=0.009)$ and zeaxanthin $(P=0.007)$ were significantly lower than first sample levels. Serum levels of carotenoids were moderately to highly reproducible within subjects, and the number of days of measurement necessary to rank individuals correctly according to their long-term carotenoid levels was close to one for alphacarotene, beta-carotene, lutein and alpha-tocopherol (Table 1).

\section{Relation to dietary intake}

Serum carotenoids except lycopene correlated positively with estimated dietary intake (FFQ) of fruits and vegetables, after correction for BMI (Table 2). They also correlated positively with dietary intake of cereals and cereal products, fish and dairy products, and negatively with consumption of meat and meat products, such as cured meat and gravy. Serum lycopene was not significantly correlated with any food group. Correlation coefficients were generally low.

BMI was negatively associated with serum carotenoids except for lycopene. Spearman rank-order correlation coefficients varied from -0.17 for beta-carotene to -0.25 for beta-cryptoxanthin in the total population $(N=302)$ and from -0.18 for beta-carotene to -0.28 for lutein in 
Table 2 Spearman rank-order correlation coefficients between serum carotenoid concentrations and intake of selected food groups estimated by food-frequency questionnaire. Results are adjusted for body mass index $(N=302)$

\begin{tabular}{|c|c|c|c|c|c|}
\hline & $\beta$-Carotene & $\alpha$-Carotene & Lutein & Zeaxanthin & $\beta$-Cryptoxanthin \\
\hline Fruits & $0.28^{\star *}$ & $0.28^{\star *}$ & $0.21^{\star \star}$ & & $0.39^{\star \star}$ \\
\hline Fruit juices & $-0.15^{\star \star}$ & & & & $0.13^{\star}$ \\
\hline Vegetables & $0.19^{* *}$ & $0.24^{\star \star}$ & $0.22^{\star \star}$ & & $0.24^{\star *}$ \\
\hline Cereals & $0.16^{\star *}$ & $0.15^{\star \star}$ & & $0.15^{\star \star}$ & $0.15^{\star \star}$ \\
\hline Fish & $0.20^{\star *}$ & $0.23^{\star \star}$ & $0.12^{\star}$ & & $0.12^{*}$ \\
\hline Poultry & & & $0.16^{\star *}$ & & \\
\hline Dairy products & & $0.12^{\star}$ & & & $0.22^{* *}$ \\
\hline Meat & $-0.14^{\star}$ & $-0.13^{*}$ & & & \\
\hline Cured meat & $-0.21^{\star \star}$ & $-0.14^{\star}$ & $-0.16^{\star \star}$ & & $-0.17^{\star \star}$ \\
\hline Gravy & $-0.23^{\star \star}$ & $-0.17^{\star \star}$ & & & \\
\hline Tomato dishes $†$ & & & & $-0.14^{*}$ & \\
\hline
\end{tabular}

† Pasta with tomato sauce, pizza.

* $P<0.05$.

** $P<0.01$.

the subgroup of 228 subjects. In that subgroup, serum cholesterol was weakly correlated with serum betacarotene $(r=0.16)$, lutein $(r=0.19)$, zeaxanthin $(r=$ $0.34)$, beta-cryptoxanthin $(r=0.20)$ and lycopene $(r=$ $0.22)$, as was serum triglycerides with alpha-carotene $(r=-0.16)$, beta-carotene $(r=-0.18)$, lutein $(r=$ $-0.15)$ and beta-cryptoxanthin $(r=-0.15)$.

Except for the concentration of beta-carotene that was predicted by both fruit and vegetable consumption, serum carotenoids were either predicted by fruit consumption (alpha-carotene and beta-cryptoxanthin) or by vegetable consumption (lutein, zeaxanthin and lycopene) (Table 3). Serum cholesterol was predictive for serum carotenoids except for beta-cryptoxanthin, and serum triglycerides for carotenoids except zeaxanthin and lycopene.

Correction for serum cholesterol, serum triglycerides or correction for both blood lipids simultaneously in addition to correction for BMI did not change correlations between estimated intake of fruits and serum carotenoids
(Table 4). Correlations with vegetable consumption were slightly higher after additional adjustment for serum cholesterol and triglycerides.

\section{Discussion}

The usefulness of serum carotenoids as biomarkers of fruit and vegetable consumption in epidemiological studies depends on their reproducibility, their relation with intake, and the influence of other endogenous and exogenous parameters on serum concentrations. The ability to rank subjects adequately according to their usual level based on one single blood measurement is highly important for large-scale epidemiological studies, where repeat blood sampling and analysis are often not feasible.

The moderate to high reproducibility of serum carotenoids found in this study in a group of women participating in the New York Women's Health Study corresponds to what has been found in other studies ${ }^{14-17}$.

Table 3 Multiple regression analysis $(N=228)$

\begin{tabular}{|c|c|c|c|c|c|c|c|c|c|c|c|c|c|}
\hline \multirow{3}{*}{$\begin{array}{l}R \text {-square model } \\
\text { Variable }\end{array}$} & \multirow{3}{*}{$\frac{d f}{15}$} & \multirow{2}{*}{\multicolumn{2}{|c|}{$\begin{array}{c}\text { Beta-carotene } \\
0.241\end{array}$}} & \multirow{2}{*}{\multicolumn{2}{|c|}{$\begin{array}{c}\text { Alpha-carotene } \\
0.207\end{array}$}} & \multirow{2}{*}{\multicolumn{2}{|c|}{$\begin{array}{l}\text { Lutein } \\
0.264\end{array}$}} & \multirow{2}{*}{\multicolumn{2}{|c|}{$\begin{array}{c}\text { Zeaxanthin } \\
0.317\end{array}$}} & \multirow{2}{*}{\multicolumn{2}{|c|}{$\begin{array}{c}\text { Beta-cryptoxanthin } \\
0.262\end{array}$}} & \multirow{2}{*}{\multicolumn{2}{|c|}{$\begin{array}{c}\text { Lycopene } \\
0.176\end{array}$}} \\
\hline & & & & & & & & & & & & & \\
\hline & & rSS† & $P$-value $\ddagger$ & rSS & $P$-value & rSS & $P$-value & rSS & $P$-value & rSS & $P$-value & rSS & $P$-value \\
\hline Fruit§ & 1 & 10.7 & 0.0079 & 13.5 & 0.0069 & & & & & 10.2 & 0.0060 & & \\
\hline Vegetable§ & 1 & 7.0 & 0.0311 & & & 12.7 & 0.0021 & 4.5 & 0.0365 & & & 11.3 & 0.0250 \\
\hline Cereal§ & 1 & & & & & 15.4 & 0.0008 & 11.9 & 0.0007 & 5.3 & 0.0468 & & \\
\hline Tomato dishes§ণ & 1 & & & & & & & 12.4 & 0.0006 & & & & \\
\hline Potatoes§ & 1 & & & & & 7.1 & 0.0213 & & & & & & \\
\hline Cured meat§ & 1 & & & & & & & 5.7 & 0.0191 & & & & \\
\hline Menstrual status & 1 & & & & & & & 4.1 & 0.0445 & & & & \\
\hline BMI & 1 & & & & & 19.8 & 0.0001 & 14.8 & 0.0002 & 11.2 & 0.0040 & & \\
\hline Cholesterol & 1 & 9.9 & 0.0105 & 11.5 & 0.0127 & 13.4 & 0.0016 & 26.1 & $<0.0001$ & & & 41.4 & $<0.0001$ \\
\hline Triglycerides & 1 & 13.6 & 0.0028 & 11.2 & 0.0137 & 6.2 & 0.0303 & & & 10.1 & 0.0063 & & \\
\hline Season & 3 & 12.0 & 0.0465 & & & & & & & & & & \\
\hline Date of first visit & 1 & & & 7.6 & 0.0418 & & & & & & & & \\
\hline
\end{tabular}

† Ratio of variable type III sum of squares to the model sum of squares, multiplied by 100 .

$\ddagger P$-values of $F$-test on type III sum of squares estimate.

$\S$ Estimated intake.

I) Pasta with tomato sauce, pizza, etc.

df: degrees of freedom. 
Table 4 Spearman rank-order correlation coefficient between estimated consumption of fruit or vegetables and serum carotenoid concentrations as a function of adjustment parameters $(N=228)$

\begin{tabular}{|c|c|c|c|c|c|}
\hline & Adjustment & Beta-carotene & Alpha-carotene & Lutein & Beta-cryptoxanthin \\
\hline \multicolumn{6}{|l|}{ Fruit } \\
\hline & BMI & 0.30 & 0.23 & 0.25 & 0.40 \\
\hline & BMI, Chol & 0.28 & 0.22 & 0.22 & 0.38 \\
\hline & BMI, Trig & 0.32 & 0.25 & 0.26 & 0.41 \\
\hline & BMI, Chol, Trig & 0.31 & 0.23 & 0.24 & 0.40 \\
\hline \multicolumn{6}{|l|}{ Vegetable } \\
\hline & BMI & 0.22 & 0.25 & 0.27 & 0.26 \\
\hline & BMI, Chol & 0.21 & 0.24 & 0.26 & 0.26 \\
\hline & BMI, Trig & 0.24 & 0.27 & 0.28 & 0.28 \\
\hline & BMI, Chol, Trig & 0.25 & 0.27 & 0.29 & 0.29 \\
\hline
\end{tabular}

Beta-carotene appears to be highly reproducible and a single sample is necessary to rank individuals accurately according to their medium- to long-term level ${ }^{6,7}$. For the other serum carotenoids, the necessary number of repeat samples diverges, possibly because of population-specific ratios of within- to between-subject variability as a function of population-specific dietary patterns. Nevertheless, these figures should be interpreted with caution, as calculations of within-subject variation were based on only three repeat measurements from 48 subjects in this study. In addition, the effect of season could not be tested, as repeat samples were collected at almost exactly one-year intervals. For beta-carotene, reproducibility in our study was comparable to results from other studies that included seasonal variation, which may indicate a limited influence of season in our study population.

A stable dietary pattern may explain the high reproducibility of serum carotenoids, as carotenoid-containing foods and supplements are the only source for humans. Homeostatic control of carotenoid concentrations has not been described, but subjects on a beta-carotene-restricted diet were found to maintain initial beta-carotene concentrations over several days or weeks ${ }^{18}$. The response to an oral dose of beta-carotene (either as a supplement or in food) was found to vary widely among subjects ${ }^{19}$, possibly as results of variable levels of mucosal uptake and excretion. High between-subject variability of serum carotenoid concentrations together with low withinsubject variability results in high reproducibility.

Consumption of fruit and vegetables is often part of a healthy food pattern, replacing the consumption of less healthy foods. This was confirmed in our study, where foods that contain few carotenoids but that are probably associated with a healthy food pattern (cereals, fish) were positively associated with serum carotenoids whereas meat and meat products were negatively associated. Serum lycopene was not significantly associated with dietary intake of fruit and vegetables. Lycopene is derived primarily from tomatoes and tomato products. Consumption of more lycopene-rich foods such as pizza, ketchup, spaghetti and chilli does not necessarily coincide with high fruit and vegetable consumption.
Correlations between intake of fruit and vegetables and blood carotenoid concentrations rarely exceed $r=0.5^{20}$, as was found in our study. High individual day-to-day variation in blood levels, as suggested by Tangney et al. ${ }^{7}$, does not seem to explain the low correlation, as reproducibility of blood carotenoid concentrations is high. Errors in estimates of dietary intake with foodfrequency questionnaires might in part explain the low correlation. Other possible explanations may be factors related to absorption, post-absorptive metabolism or even physiological regulation of nutrient levels, which can be important determinants of variation in the marker independently of the true intake level. If the effects of such intervening factors vary systematically between individuals, this will affect the validity of the marker as a measurement of dietary intake level, and correlations with questionnaire measurements may remain weak even if the average of several replicate biomarker measurements is taken for each individual ${ }^{20,21}$.

In multiple regression analysis, fruit consumption was a predictor for certain serum carotenoids while others were predicted by intake of vegetables. In spite of its lower reproducibility, serum beta-cryptoxanthin correlated more strongly with estimated intake of fruits than other serum carotenoid concentrations. This can be explained by the relative predominance of, for example, beta-cryptoxanthin in fruits and lutein in vegetables, and underlines the importance of measuring several carotenoids in blood to evaluate fruit and vegetable consumption.

The weak negative association of BMI with serum carotenoids has been found in other studies ${ }^{16,22}$ but has not yet been clearly explained. BMI often correlates positively with blood levels of triglycerides but it is not known if the first is a result of the second or vice versa.

Although serum cholesterol and triglycerides were predictors of serum carotenoid concentrations and were correlated (weakly) with serum carotenoids ${ }^{22}$, adjustment for their levels did not the change rank-order correlation with dietary intake of fruit and only slightly increased that with intake of vegetables. As cholesterol and triglyceride levels were highly reproducible, reproducibility of serum 
carotenoids was not modified by adjustment, neither were estimates of relative risk of serum carotenoids for breast cancer in the same population ${ }^{10}$. Carroll et al. ${ }^{14}$ found no effect from cholesterol and triglycerides adjustment either.

The relation between carotenoids and blood levels of cholesterol and triglycerides is not well understood. Carotenoids appear to be absorbed by duodenal mucosal cells by a mechanism involving passive diffusion, similar to that of cholesterol and the products of triglyceride lipolysis ${ }^{23}$. Most of the cholesterol circulating in blood is, however, of endogenous origin, so common absorption seems an unlikely explanation for correlated blood levels. The relation is more probably a result of common transport in blood. Carotenoids are transported in blood by lipoproteins, as are cholesterol and triglycerides. The distribution among the various lipoprotein classes appears to be determined by the physical characteristics of individual carotenoids and the lipid composition of the lipoproteins $^{23}$. As adjustment for cholesterol and triglycerides does not modify the relations of serum carotenoids to diet or disease, and as their concentrations do not therefore seem to be important determining factors, adjustment of serum or plasma carotenoid concentrations for these blood lipids does not seem justified.

In this group of women from New York, although one single serum measurement can accurately rank subjects according to the usual serum level for alpha-carotene, beta-carotene and lutein, serum carotenoid concentrations correlated only moderately with estimated consumption of fruit or vegetables. As this study cannot estimate how much of the low correlation between diet and serum concentrations is explained by errors in the dietary questionnaire and how much by determinants of serum concentrations other than fruit and vegetable consumption, serum carotenoids should be used with caution as biomarkers of fruit and vegetable consumption.

\section{Acknowledgements}

This work was supported by grants CA-34588, CA-13343 and CA-16087 from the National Cancer Institute, grant ES-00260 from the National Institute of Environmental Health Sciences and by the Europe Against Cancer Programme of the EU. The authors thank all women who generously volunteered for the study and are grateful to Béatrice Vozar and David Achaintre for their assistance. Anne Linda van Kappel received funding from APRIFEL, France. Echinenon as internal standard for HPLC analysis was a gift from Hoffman La Roche, Basle, Switzerland.

\section{References}

1 World Cancer Research Fund/American Institute for Cancer Research. Vegetables and fruits. In Food, Nutrition and the Prevention of Cancer: A Global Perspective. Washington, DC:
World Cancer Research Fund/American Institute for Cancer Research, 2000; 436-46.

2 Pfander H. Key to Carotenoids. Basel: Birkhauser Verlag, 1987.

3 Kull D, Pfander H. List of new carotenoids. In: Britton G, Liaaen-Jensen S, Pfander H, eds. Carotenoids. Basel: Birkhauser Verlag, 1995; 295-317.

4 Khachik F, Spangler CJ, Smith JC Jr, Canfield LM, Steck A, Pfander $\mathrm{H}$. Identification, quantification, and relative concentrations of carotenoids and their metabolites in human milk and serum. Anal. Chem. 1997; 69: 1873-81.

5 Khachik F, Beecher GR, Goli MB, Lusby WR, Smith JC Jr. Separation and identification of carotenoids and their oxidation products in the extracts of human plasma. Anal. Chem. 1992; 64: 2111-22.

6 Cooney RV, Franke AA, Hankin JH. et al. Seasonal variations in plasma micronutrients and antioxidants. Cancer Epidemiol. Biomarkers Prev. 1995; 4: 207-15.

7 Tangney CC, Shekelle RB, Raynor W, Gale M, Betz EP. Intraand interindividual variation in measurements of betacarotene, retinol, and tocopherols in diet and plasma. Am. J. Clin. Nutr. 1987; 45: 764-9.

8 Toniolo PG, Pasternack BS, Shore RE. et al. Endogenous hormones and breast cancer: a prospective cohort study [see comments]. Breast Cancer Res. Treat. 1991; 18(Suppl. 1): S23-6.

9 Toniolo PG, Levitz M, Zeleniuch-Jacquotte A. et al. A prospective study of endogenous estrogens and breast cancer in postmenopausal women [see comments]. J. Natl. Cancer Inst. 1995; 87: 190-7.

10 Toniolo P, Van Kappel AL, Akhmedkhanov A. et al. Serum carotenoids and breast cancer. Am. J. Epidemiol. 2000; (in press).

11 Steghens JP, Van Kappel AL, Riboli E, Collombel C. Simultaneous measurement of seven carotenoids, retinol and alpha-tocopherol in serum by high-performance liquid chromatography. J. Chromatogr. B. Biomed. Sci. Appl. 1997; 694: 71-81.

12 Block G, Hartman AM, Dresser CM, Carroll MD, Gannon J, Gardner L. A data-based approach to diet questionnaire design and testing. Am. J. Epidemiol. 1986; 124: 453-69.

13 Nelson M, Black AE, Morris JA, Cole TJ. Between- and within-subject variation in nutrient intake from infancy to old age: estimating the number of days required to rank dietary intakes with desired precision. Am. J. Clin. Nutr. 1989; 50 155-67.

14 Carroll YL, Corridan BM, Morrissey PA. Carotenoids in young and elderly healthy humans: dietary intakes, biochemical status and diet-plasma relationships. Eur. J. Clin. Nutr. 1999; 53: 644-53.

15 Campbell DR, Gross MD, Martini MC, Grandits GA, Slavin JL, Potter JD. Plasma carotenoids as biomarkers of vegetable and fruit intake. Cancer Epidemiol. Biomarkers Prev. 1994; 3 493-500.

16 Scott KJ, Thurnham DI, Hart DJ, Bingham SA, Day K. The correlation between the intake of lutein, lycopene and betacarotene from vegetables and fruits, and blood plasma concentrations in a group of women aged 50-65 years in the UK. Br. J. Nutr. 1996; 75: 409-18.

17 Potischman N, Byers T, Houghton L, Root M, Nemoto T, Campbell TC. Effects of breast cancer treatments on plasma nutrient levels: implications for epidemiological studies. Cancer Epidemiol. Biomarkers Prev. 1992; 1: 555-9.

18 Johnson EJ, Russell RM. Distribution of orally administered beta-carotene among lipoproteins in healthy men. Am. J. Clin. Nutr. 1992; 56: 128-35.

19 Brown ED, Micozzi MS, Craft NE. et al. Plasma carotenoids in normal men after a single ingestion of vegetables or purified beta-carotene. Am. J. Clin. Nutr. 1989; 49: 1258-65.

20 Kaaks R, Riboli E, Sinha R. Biochemical markers of dietary 
intake. In: Toniolo P, Boffetta P, Shuker DEG, Rothman N Hulka B, Pearce N, eds. Application of Biomarkers in Cancer Epidemiology. Lyon: International Agency for Research on Cancer, 1997; 103-26.

21 van't Veer, Kardinaal AF, Bausch-Goldbohm RA, Kok FJ.

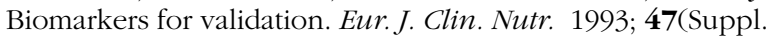
2): $558-63$.
22 Brady WE, Mares-Perlman JA, Bowen P, Stacewicz-Sapuntzakis M. Human serum carotenoid concentrations are related to physiologic and lifestyle factors. J. Nutr. 1996; 126: 129-37.

23 Parker RS, Absorption, metabolism, and transport of carotenoids. FASEB J. 1996; 10: 542-51. 